\title{
Gestión académica saludable en el contexto universitario
}

\author{
Healthy Academic Processes in the University Context
}

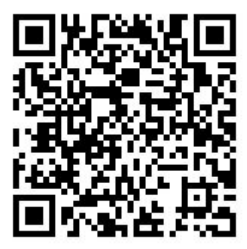

\author{
Ileana Castillo-Cedeño ${ }^{1}$ \\ Universidad Nacional \\ Centro de Investigación y Docencia en Educación \\ Heredia, Costa Rica \\ ileana.castillo.cedeno@una.cr \\ Luz Emilia Flores-Davis ${ }^{2}$ \\ Universidad Nacional \\ Rectoría Adjunta \\ Heredia, Costa Rica \\ luz.flores.davis@una.cr \\ Giselle Miranda-Cervantes ${ }^{3}$ \\ Universidad Nacional \\ Centro de Investigación y Docencia en Educación \\ Heredia, Costa Rica \\ giselle.miranda.cervantes@una.cr
}

Recibido 13 de marzo de 2015 • Corregido 8 de agosto de 2015 • Aceptado 14 de agosto de 2015

\begin{abstract}
${ }^{1}$ Doctora en Educación con especialidad en Mediación Pedagógica. Egresada del Doctorado en Diseño Curricular y Evaluación Educativa de la Universidad de Valladolid, España. Magíster en Psicopedagogía. Magíster en Educación Preescolar. Especialista en didáctica de la educación del consumidor. Universidad de Valladolid, España. Profesora, bachiller y licenciada en Ciencias de la Educación con énfasis en Educación Preescolar, Universidad Nacional de Costa Rica. Fue vicedecana del Centro de Investigación y Docencia en Educación durante el período 2008 al 2013. Fue asesora de la Vicerrectoría Académica en los programas de diseño y gestión curricular y en el programa de Evaluación Académica y Desarrollo profesional. En el 2013 participó como candidata a Vicerrectora Académica de la Universidad Nacional de Costa Rica. Actualmente se desempeña como investigadora y ocupa el cargo de Decana del Centro de Investigación y Docencia en Educación de la Universidad Nacional de Costa Rica. Autora de varias publicaciones sobre educación, pedagogía, diversidad, liderazgo, educación superior en revistas de su especialidad.

${ }^{2}$ Doctora en Educación con especialidad en Mediación Pedagógica. Máster en Educación Superior. Catedrática universitaria. Ha ocupado los cargos de directora de Docencia, directora de Desarrollo Profesional y Cooperación Universitaria, presidenta de la Junta de Becas y Coordinadora de la Comisión Técnica de Admisión de la Universidad Nacional. Se desempeña como investigadora, extensionista y docente en la División de Educación Básica del Centro de Investigación y Docencia en Educación de la Universidad Nacional, Costa Rica. Actualmente es la Rectora Adjunta de la Universidad Nacional. Entre sus publicaciones más recientes destacan trabajos para la alfabetización de personas adultas.

${ }^{3}$ Doctora en Educación con especialidad en Mediación Pedagógica. Bachiller, licenciada y máster en Psicología de la Universidad de Costa Rica. Labora como académica e investigadora desde 1990 en la División de Educación Básica del Centro de Investigación y Docencia en Educación (CIDE) de la Universidad Nacional de Costa Rica, unidad académica en la que ocupó los cargos de directora, miembro del Consejo Académico y coordinadora de las carreras de Educación Especial y de Enseñanza del Inglés para I y ll ciclos. Su interés en el campo de la investigación destaca las áreas de diversidad, seguimiento a egresadas de la carrera de Educación Especial, relaciones interpersonales, clima social de aula y pedagogía saludable.
\end{abstract}


doi: http://dx.doi.org/10.15359/ree.19-3.2

URL: http://www.una.ac.cr/educare

CORREO: educare@una.cr

Resumen. El presente artículo científico tiene como objetivo identificar las percepciones con respecto a los procesos de gestión académica saludable en el contexto universitario. Aporte gracias a los procesos de investigación socioeducativa que se generan en la Universidad Nacional de Costa Rica (UNA), en el Centro de Investigación y Docencia en Educación (CIDE). El tema de la salud es parte del plan institucional y del Centro. Considerando que la salud, al igual que la educación, es un derecho humano fundamental que amerita responsabilidades desde la pedagogía como ciencia de la educación que investiga, analiza y transforma, es urgente, de cara a los nuevos desafíos mundiales que ponen en evidencia las crisis planetarias vinculadas a la salud, atenderlo. La investigación se fundamenta en el paradigma naturalista y una metodología que asume un tipo de investigación mixta, donde se contrastan datos cualitativos y cualitativos a partir de la utilización de entrevista semiestructurada para lograr un análisis más profundo que permite contrastar percepciones, teorías y prácticas. De los resultados de impacto se tiene que es inédito el concepto pedagogía saludable en el contexto universitario. La vinculación entre pedagogía y salud como un constructo teórico, epistemológico, axiológico holístico que contempla la teoría de la complejidad permite a la universidad asumir desafíos con sus enormes potencialidades; promover ambientes, estilos, organizaciones saludables desde la gestión académica desde la vertiente individual y colectiva. Es posible la construcción de nuevos órdenes de sentido que asumen de manera corresponsable la redignificación de la vida universitaria en sus diversos espacios y dimensiones. La investigación tiene el valioso potencial de convertirse en un elemento dinamizador de políticas institucionales que favorecen la vida.

Palabras claves. Gestión académica, pedagogía saludable, educación superior.

Abstract. This scientific article aims to identify the perceptions of healthy academic administrative processes in the university context. This contribution was directed by socio-educational research processes generated at the National University of Costa Rica (UNA), in the Center for Research and Teaching in Education (CIDE). The issue of health is part of the institutional plan and the Center. Whereas health, like education, is a fundamental human right that deserves responsibilities from pedagogy as educational science research, which analyzes and transforms. Therefore, it is urgent, in the face of new global challenges to address planetary crises linked to health. This research is based on the naturalistic paradigm and a methodology that assumes a type of joint research, where using a semi-structured interview achieves a deeper analysis that allows contrasting perceptions, theories and practices by comparing qualitative and quantitative data. From the results of impact the concept of Healthy Pedagogy is unknown in the university context. The connection between education and health as a holistic theoretical, epistemological and axiological construction, it includes the complexity theory that allows the university to take challenges with an enormous potential; promoting environments, styles and healthy organizations from healthy academic administrations from both individual and collective aspects. It is possible to construct new sense of orders, which assume in a responsible manner to re-dignify the university life in its various spaces and dimensions. Research has the valuable potential to become a dynamic element of institutional policies in favor of life.

Keywords. Academic administration, healthy pedagogy, higher education. 


\section{Antecedentes}

El presente artículo de investigación ha sido sustentado a partir del proceso investigativo llevado a cabo en el Centro de Investigación y Docencia en Educación (CIDE) de la Universidad Nacional de Costa Rica (UNA).

El Proyecto Pedagogía Saludable fue inscrito en el año 2013 (I. Castillo, R. Castillo y Miranda, 2013) con una vigencia de tres años, es decir, hasta el 2015. Es un proyecto de tipo interdisciplinario inscrito en la universidad dentro del subprograma de investigación.

El equipo de investigadoras fundamentadas en que la salud y la educación constituyen derechos inalienables, que deben ser correlacionados para tener una visión más integradora y holística, y desde el paradigma del pensamiento complejo, asumen y comprenden que las instituciones de educación superior tienen una responsabilidad compartida de asumir el reto de mejorar la calidad de vida en su máxima expresión. Las investigadoras asumen el proyecto con la claridad de que la pedagogía, más allá de un constructo teórico, es ciencia que investiga y se proyecta para generar transformaciones que mejoren la calidad de vida de las personas y otros seres vivos en sus diversos entornos de convivencia. Una visión unificada de lo que implica mente, materia y vida.

\section{Justificación}

Este artículo parte de la comprensión de la trama de relaciones sociales, económicas, políticas, culturales que han generado nuevas y necesarias reflexiones en el ámbito de la atención de la salud y la pedagogía. La salud constituye una problemática que necesariamente, desde una visión que comprende la complejidad de la vida, asume la necesidad de diálogo interdisciplinar, para mirar el contexto actual de la salud y de la vida como un ejercicio consciente que se asume no solo desde la parte médica, sino desde el conocimiento pedagógico, que permite educar desde el conocimiento de que la pedagogía es ciencia de la vida. Por lo tanto, se liga directamente con la salud, la cual constituye, además de una necesidad vital, una responsabilidad compartida entre personas y grupos que valoran las nuevas tendencias mundiales que exigen atención sostenida y decidida para prevenir y atacar las situaciones de salud que viven las poblaciones y el planeta en general.

El artículoalude, de forma general, referencias bibliográficasfundamentales, con la convicción de que se ha generado a partir de la lectura de abundante bibliografía, conocimiento que permite comunicar planteamientos interconectados que no precisan hacer alusión demandante de lo escrito por otros autores. Creemos en la necesidad y posibilidad de generar conocimiento propio y proponer nuevas construcciones desde la experiencia y la investigación realizada. 
doi: http://dx.doi.org/10.15359/ree.19-3.2

URL: http://www.una.ac.cr/educare

CORREO: educare@una.cr

\section{Marco referencial}

En el marco del paradigma emergente diversos autores (Assmann, 2002; Gutiérrez, 1981; Maturana, 1999; Maturana y Varela, 1986; Morin, 2001) han desarrollado propuestas que impactan la pedagogía en diferentes dimensiones desde una visión trasformadora. Por otra parte, la salud también está reconceptualizándose con el aporte de autores como Dossey (2004), Payán (2000) y Najmanovich (2001), entre otros. Ello nos permite, a las investigadoras, establecer la relación dialógica entre pedagogía y salud. Nos otorgamos el mérito de desarrollar un constructo téorico, epistemológico y axiológico innovador denominado "pedagogía saludable".

Desde esta perspectiva, la pedagogía saludable se caracteriza por ser una pedagogía holística y compleja que favorece la convivencia, la cooperación, la expresión y la creación. Es una pedagogía de la libertad, la democracia, la responsabilidad y el cuidado. Es una experiencia gratificante que se construye en comunidad aprendiente. (I. Castillo, R. Castillo, Flores y Miranda, 2014, p. 317)

La salud, la educación y la vida misma se han asumido, tradicionalmente, desde el paradigma mecanicista transitando un camino de supuesta certidumbre. No obstante, se han empezado a recorrer nuevos rumbos y a resignificarse la libertad y la esperanza para reencontrar el puente que conecta la salud con la vida y la vida con la salud. El mundo está cambiando de manera acelerada y el viejo paradigma se resquebraja.

El inicio del siglo XXI ha forjado nuevas tendencias paradigmáticas y un ensanchamiento de la conciencia cósmica y planetaria con fundamento en los avances del conocimiento, principalmente en tres campos de la ciencia, a saber: la física -con el descubrimiento de las leyes básicas, al desentrañar los secretos del átomo-, la informática -al progresar en el desarrollo del ordenador electrónico-, y la biología -al descodificar el núcleo de la célula y descubrir el ADN-. Todo ello genera visiones alternativas en todos los campos del saber e impacta nuestra vida cotidiana.

Es así como surge el paradigma emergente como opción que posibilita una nueva manera de pensar, de sentir y de actuar; un modo diferente de vivir que nos invita a confrontarnos con nosotras mismas o con nosotros mismos, con nuestro propio yo, nuestro entorno y con la realidad que hemos creado; una ética que nos une no solo entre seres humanos, sino con el resto de la naturaleza.

También nos invita a construir una cultura respetuosa de la vida. Esta nueva concepción se caracteriza por su visión ecológica, holista y compleja. En ella tiene cabida una nueva visión de la educación y de la salud; se despliegan nuevas maneras de entender las relaciones y los procesos vitales. 
En el nuevo paradigma se entiende que la enfermedad "...no es lo contrario de la salud sino el proceso vital por el cual ese ser, compuesto de mente y cuerpo como unidad, busca y mantiene la armonía con él mismo y con su entorno" (Payán, 2000, p. 4). Esta es una perspectiva que restituye la libertad al ser humano, al hacerle tomar conciencia de su poder vital como ser activo y dinámico; y funda una relación de solidaridad donde la salud se concibe como la "armonía resultante de una fluidez de movimiento" (Dossey, 1999, p. 13).

En la salud este enfoque tiene connotaciones de gran importancia, dado que en la medicina ortodoxa, si bien la especialización ha logrado avances cardinales, muchas veces se ha dejado de lado la comprensión del ser humano como ser integral.

Hoy sabemos que los pensamientos y las emociones pueden influir en la cura tanto de manera positiva como negativa, y ello en nuestros cuerpos y en los cuerpos de otras y otros, aún a la distancia (Dossey, 2004). Este hallazgo implica reconocer la importancia de la participación plena de cada persona en sus propios procesos vitales, así como la necesidad de superar la estandarización característica de la cultura tradicional.

Aprender a vivir plenamente, sanamente, es un reto para la educación actual. Asimismo, es necesario tomar en cuenta que la sensación de prisa, propia de nuestra cultura, trae consigo una gran cantidad de problemas físicos, "la ansiedad, el estrés y la tensión figuran entre los factores que generan hipertensión, arteriosclerosis y enfermedades cardíacas en general, es decir, algunas de las causas de mortandad más frecuentes en nuestra sociedad" (Dossey, 1999, pp. 255-256). Una vida en salud pasa también por estados serenos, relajados, gratos, los cuales impactan los niveles hormonales en la sangre, el ritmo cardíaco, la presión y el flujo sanguíneo, entre otros. Y en este sentido, la pedagógica tiene un papel de gran relevancia en la formación de personas y sociedades que aprendan desde una relacionalidad armoniosa.

Lo anterior evidentemente introduce la reflexión de que la salud integra incomparables ámbitos de acción en los cuales las personas se desarrollan e interactúan y, por tanto, es quehacer de la pedagogía reflexionar y accionar, en función de la vida, dado que estamos viviendo un cambio paradigmático a nivel planetario: se revela la complejidad que emerge de la existencia, aspecto que implica el reconocimiento de que la realidad tiene componentes diversos e inciertos que deben abordarse de manera holística desde todos los ámbitos del desarrollo humano. Este cambio, además, conlleva una ruptura con el modelo de organización y gestión tradicional y exige crecer a partir de la suma de esfuerzos y voluntades de las personas en sus entornos cotidianos.

La educación es un derecho fundamental que debe caminar de la mano del desarrollo humano para alcanzar mejores niveles de vida. Surge, entonces, un reto importante y necesario de abordar desde la pedagogía, para responder a una época que exige plantear los procesos en que se da la gestión académica, como ese espacio de interrelación en la reflexión y acción y para estas, en función de organizaciones de vida más saludables. 
doi: http://dx.doi.org/10.15359/ree.19-3.2

URL: http://www.una.ac.cr/educare

CORREO: educare@una.cr

Una organización saludable, según Gimeno, Grandío, Marqués (2013), tiene como característica:

...promovery potenciar la sinergia positiva entre su propio crecimiento, el de sus miembros y el de la comunidad. La construcción de este tipo de organizaciones se ha convertido en uno de los desafíos del mundo organizacional y, por ende, de la propia sociedad. Su viabilidad y concreción pueden determinar, en buena medida, el modelo social del futuro a mediano y largo plazo. (p. 41)

De lo anterior se desprende que la ineludible tarea pedagógica de las universidades es la de fomentar -en su interior, como organizaciones vivas- la comprensión de que los cambios sociales exigen mayores niveles de investigación y acción para el bienestar personal y social placentero.

\section{Gestión académica saludable en y desde la cotidianeidad}

Las universidades son organismos vivos dedicados a generar dinámicas de entendimiento y comprensión del contexto global para mejorar la calidad de vida de las personas. De ahí que se indique que, como organismos vivos, tienen una función socioeducativa innovadora y difusora de vida.

El clima organizacional se basa en las percepciones individuales, a menudo se define como los patrones recurrentes de comportamiento, actitudes y sentimientos que caracterizan la vida en la organización, y se refieren a las situaciones actuales en una organización y los vínculos entre los grupos de trabajo, los empleados y el desempeño laboral. (Domínguez, Ramírez, García, 2013. p. 62)

El respeto a ese conjunto de percepciones individuales y la capacidad de mediar para lograr condiciones de entendimiento colectivas permiten una gestión académica saludable que involucra la suma de voluntades y posibilita que las personas se aventuren y se identifiquen con proyectos pedagógicos comunes que engrandezcan a la colectividad y lleven a niveles de convivencia, identidad y bienestar, necesarios para el despliegue de una gestión académica transformadora.

Las innovaciones se caracterizan por políticas claras de motivación que cautivan a la totalidad de miembros de la comunidad educativa, quienes comprenden y se identifican plenamente con el compromiso de gestar experiencias de aprendizaje y de vida que revelen y atiendan el bienestar común. Desde esta perspectiva, la motivación supera las resistencias y los estados de comodidad. Implica una participación activa y articulada de las personas en proyectos educativos que enaltezcan y promuevan cambios en la cultura organizacional, aspecto que implica evaluación permanente.

6

Ileana Castillo-Cedeño, Luz Emilia Flores-Davis y Giselle Miranda-Cervantes (c) (1) (3) 
Desde luego, hablar de innovación educativa, asociada al clima organizacional, conlleva implícitos otros componentes tales como: aspectos de infraestructura, inversión, ambientes de confianza, de encuentro, entre otros tantos aspectos que configuran las organizaciones saludables.

Ciertamente tal y como lo afirman Vila, Escotet y Goñi (2009):

La innovación es un término que ha sido usado de diversos modos y con bastante ambigüedad. Ha sido confundido e intercambiado con otros fines. Su origen latino, innovatio, se refiere tanto al proceso como al resultado y su prefijo in equivale a en, dentro, dentro del interior. En este marco se entiende la innovación como incorporación de algo nuevo dentro de una realidad existente, en cuya virtud ésta resulta modificada. (p. 133)

Si consideramos a la organización que aprende como aquella que facilita el aprendizaje de todos sus miembros y continuamente se transforma a sí misma, estamos resaltando el valor del aprendizaje como la base fundamental de la organización. El desarrollo de la organización se basa en el desarrollo de las personas y en su capacidad para incorporar nuevas formas de hacer a la institución en las que trabajan. (Gairín, 2010, p. 22)

En este sentido, el proceso de aprender constituye el fundamento para el mejoramiento continuo de las personas en lo particulary de la institución en términos generales. La complejidad propia de una universidad autónoma que se autoorganiza, conlleva la necesidad de establecer redes de relaciones en un ambiente de solidaridad y valoración de los otros y de las otras.

La gestión académica se lleva a cabo en un espacio educativo y, por tanto, tiene que ver con la pedagogía que se fomenta. Si se pretenden formas de vida sana, es necesario que la gestión vele por el bienestar de la comunidad universitaria.

La pedagogía saludable solamente se puede explicar desde la complejidad que implican los ambientes de aprendizaje, donde las personas aprendientes y mediadoras están entrelazadas, se construyen y reconstruyen desde su individualidad y las relaciones que se establecen en todos los ámbitos y dimensiones. (I. Castillo, R. Castillo, Flores y Miranda, 2014, p. 317)

Las visiones personales sobre salud, cuidado, bienestar y calidad de vida, entre otras, tienen un impacto en la vida institucional: porque es así como la vertiente individual se entrelaza de manera recursiva con la vertiente colectiva en la construcción de sentidos y se forjan mutuamente.

La calidad de vida tiene que ver con la persona de manera integral; desde sus diversos espacios personales, familiares, comunitarios, sociales. Ardila (2003), define la calidad de vida 
doi: http://dx.doi.org/10.15359/ree.19-3.2

URL: http://www.una.ac.cr/educare

CORREO: educare@una.cr

de la siguiente manera: "Calidad de vida es un estado de satisfacción general, que surge de realizar las potencialidades que el individuo posee. Realización personal y calidad de vida son dos aspectos que se han ligado, y que sin duda están altamente correlacionados" (p. 163).

Desde una perspectiva integral e integradora, las actividades del trabajo o estudio en la universidad y el quehacer en los demás espacios personales, familiares y sociales se amalgaman junto con los valores, en la búsqueda de una vida plena y de realización personal, para otorgarle sentido a la vida.

La convivencia que se realiza en la universidad tiene que ver con su organización y con las interacciones entre los sectores académico, administrativo y estudiantil. No obstante, existen principios que caracterizan las relaciones en cada institución. Para poder hablar de una institución saludable, dichas interacciones requieren basarse en la confianza, en el respeto, en la solidaridad, en la equidad, en el diálogo. Como bien lo expresa Maturana (1996) “La confianza es el fundamento de la convivencia social, cualquiera que sea el ámbito y la multidimensionalidad de ella. De hecho, no hay substituto para la confianza en las relaciones humanas, y sin confianza no hay fenómeno social" (p. 68).

\section{Metodología}

La investigación que aquí se comparte se enmarca dentro de un paradigma cualitativo con miras a la comprensión integral y holística de la vinculación entre la vida saludable y la gestión académica universitaria, desde la mirada y el sentir de las personas que lideran esos procesos. El estudio se identifica como exploratorio (Hernández, Fernández y Baptista, 2006) y procura, a partir de una extensa gama de puntos de vista, hacer una descripción e interpretación de los hallazgos encontrados.

Por otra parte, dada la complejidad y multicausalidad de dicha vinculación, se recurrió al uso de la entrevista semiestructurada como técnica para la recolección de la información.

El grupo seleccionado para la investigación estuvo constituido por personas que, al momento de realizar la recolección de la información, se encontraban en puestos de autoridad y gestión académica en el CIDE, específicamente en las siguientes instancias: Vicedecanatura, División de Educación Básica, División de Educación para el Trabajo, División de Educación Rural, División de Educología e Instituto de Estudios Interdisciplinarios de la Niñez y la Adolescencia (INEINA). Las divisiones son instancias de especialización en el área pedagógica, también conocidas como unidades académicas o departamentos en este documento.

La entrevista utilizada se diseñó a partir de la revisión bibliográfica, fue validada mediante el criterio de jueces expertos. Se estructuró en ocho preguntas abiertas. La información fue recolectada por las investigadoras. 
Los resultados obtenidos fueron sistematizados y agrupados en categorías de análisis según las respuestas. Los datos fueron analizados desde la perspectiva paradigmática compartida por las investigadoras en concordancia con los referentes conceptuales de este artículo. De estos se desprende como información apreciable el apartado de reflexiones generales y aportes de este artículo de investigación; además de las conclusiones y construcciones relevantes de la investigación al campo del conocimiento y, por último, se resaltan los aportes de los participantes.

\section{Resultados y análisis}

Como se mencionó, se contó con la participación de todas las personas que, al momento de realizar la recolección de la información, se encontraban en puestos de autoridad y gestión académica en el CIDE. De esta manera, la población estuvo constituida por diez personas y la tabla 1 muestra la distribución correspondiente.

Tabla 1

Distribución de la población, según puesto y sexo

\begin{tabular}{|c|c|c|c|}
\hline \multirow{2}{*}{ Puesto } & \multicolumn{2}{|c|}{ Sexo } & \multirow{2}{*}{ Total } \\
\hline & Hombre & Mujer & \\
\hline Vicedecanatura & 0 & 1 & 1 \\
\hline Dirección de Unidad Académica & 4 & 1 & 5 \\
\hline Subdirección de Unidad Académica & 2 & 2 & 4 \\
\hline Total & 6 & 4 & 10 \\
\hline
\end{tabular}

A partir de la entrevista realizada a la totalidad de autoridades del CIDE, se obtuvieron los resultados que se describen a continuación.

Al indagar acerca del concepto de salud, la mayoría (90\%) evidencia una visión que involucra el bienestar integral del ser humano, haciendo alusión a componentes físicos, socioafectivos y cognitivos; solamente un (10\%) muestra una visión más localizada asociada a ausencia de enfermedad. 
doi: http://dx.doi.org/10.15359/ree.19-3.2

URL: http://www.una.ac.cr/educare

CORREO: educare@una.cr

De manera detallada, el $60 \%$ las personas del CIDE con cargos directivos, percibe la salud, como un estado o una condición de bienestar integral, el $30 \%$ lo asocia con equilibrio en diversas dimensiones. Mientras que un $10 \%$ indica que la salud es "ausencia de enfermedad". Según se muestra en la figura 1.

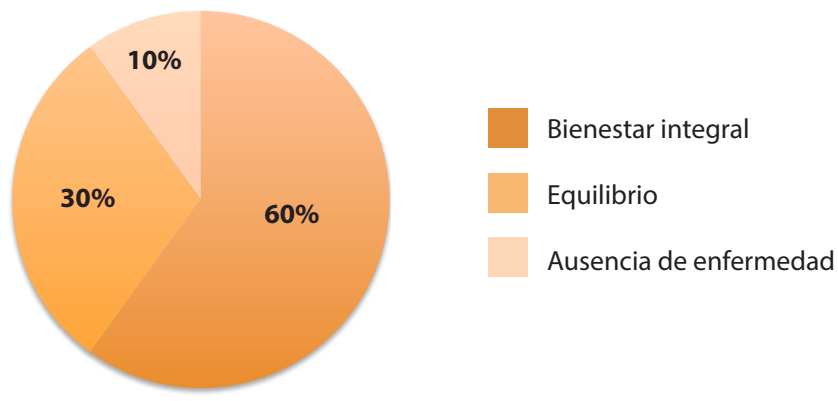

Figura 1. Concepto de salud según criterio de las autoridades del CIDE.

La figura 1 refleja que existe mayor conciencia por parte de las personas que, tal y como lo indica la Organización Mundial de la Salud (OMS) en múltiples textos, la salud no puede ser concebida solamente como ausencia de enfermedad. La salud involucra diferentes dimensiones de desarrollo del ser humano.

Algunas expresiones escritas en las entrevistas se citan porque amplían y permiten ejemplificar la forma en que se conceptualiza la salud:

a. disfrutar y gozar de la vida en términos de calidad de vida;

b. es un bienestar integral que se refleja en la parte bioquímica y física"; "la persona hoy día busca una vida que tenga sentido, no hablamos de edades sino de cómo vive su vida.

Con respecto al concepto saludable, nuevamente se obtuvo una mayoría de respuestas (90\%) que evidencia una visión que involucra aspectos donde destacan el sentido de la vida y la armonía con el ser interior; en oposición con una minoría (10\%) que se centra más en aquellas prácticas o acciones que permiten un buen funcionamiento físico (cuerpo).

La mayoría de las respuestas de las autoridades del CIDE sugiere como saludable aspectos tales como: "encontrarle sentido a la vida"; "gozar, disfrutar, tomarse el tiempo"; "gozar de la condición de salud"; "tener espacios como persona"; "la relación con otras personas y lo logrado a nivel personal, físico, emocional y en pareja"; "disfrutar de la relación en grupo"; "evitar el estrés"; y "comprometerme con lo que puedo hacer", entre otras. 
Lo anterior ratifica que, para estar saludable, es necesario conocerse a sí mismo o a sí misma y cuidarse. El amor por uno mismo o por una misma y el conocimiento permiten alejarse de las situaciones que pueden provocar malestares o enfermedades. No obstante, parece una contradicción el hecho de que cuando se les cuestiona con respecto a si se consideran una persona saludable, un $70 \%$ contesta negativamente, frente a un $30 \%$ que indica que sí se considera saludable.

Las razones que ofrecen se asocian fuertemente con asuntos laborales e inherentes al puesto que desempeñan. En la tabla 2 se destaca lo indicado.

Tabla 2

Motivos por los que se considera no saludable o saludable, según opinión de personas entrevistadas

\begin{tabular}{lc}
\hline No saludable & Porcentaje \\
\hline Enfermedades físicas y psicosomáticas asociadas al estrés laboral (colitis, migrañas, gastritis) & 10.0 \\
Condiciones de no auto-cuidado, derivadas de los horarios que impone el puesto (la falta de & 20.0 \\
horario para comer, el estrés, los problemas laborales) & 20.0 \\
Malos hábitos (tipo de alimentación, falta de sueño, llevar trabajo a la casa, fumado) & 10.0 \\
Condiciones o enfermedades crónicas (sobrepeso, metabolismo lento) & 10.0 \\
Dependencia de medicamentos & 70.0 \\
\hline Subtotal & 10.0 \\
\hline Saludable & 20.0 \\
\hline $\begin{array}{l}\text { Interés personal, motivación y toma de conciencia } \\
\text { Decisión personal aun frente a lo adverso (disfrutar la vida cuando hay salud y cuando hay } \\
\text { dolor) }\end{array}$ \\
\hline Subtotal & 30.0 \\
\hline Total & 100.0 \\
\hline
\end{tabular}

La tabla 2 evidencia que la salud tiene que ver decididamente no solo con la forma en que se concibe esta en un sentido teórico, sino cómo se vive y cómo se es capaz de equilibrar lo que se dice y lo que se hace; binomio muy usual dentro de la vida del ser humano.

Del discurso a la acción hay elementos evidentemente complejos que llevan a la contradicción. De ahí la importancia de evidenciar esas inconsistencias para ir generando actuaciones y prácticas de vida consecuentes con lo que se expresa. 
doi: http://dx.doi.org/10.15359/ree.19-3.2

URL: http://www.una.ac.cr/educare

CORREO: educare@una.cr

Aunado a las anteriores interrogantes, y considerando el cargo de gestión académica que ejercen las personas entrevistadas, se les preguntó cuán saludable considera que es su puesto en la Universidad Nacional. Las respuestas muestran una distribución similar a la obtenida a partir de la pregunta anterior, ya que la mayoría (70 \%) considera que el puesto no es saludable y el restante (30\%) opina que sí es saludable. Los motivos de su respuesta se muestran en la tabla 3.

Tabla 3

Motivos por los que el puesto se considera no saludable o saludable, según opinión de personas entrevistadas

\begin{tabular}{|c|c|}
\hline No saludable & Porcentaje \\
\hline Por el estrés que genera el puesto, hay que ser multitareas & 10.0 \\
\hline Hay recarga de tareas que obliga a llevarse trabajo a la casa & 30.0 \\
\hline Falta espacio para el crecimiento personal & 10.0 \\
\hline Pérdida de rumbo institucional y falta de establecimiento de prioridades & 10.0 \\
\hline Procesos burocráticos & 10.0 \\
\hline Subtotal & 70.0 \\
\hline \multicolumn{2}{|l|}{ Saludable } \\
\hline Actitud y manejo con que se asuma las prioridades y las responsabilidades inherentes al puesto & 30.0 \\
\hline Subtotal & 30.0 \\
\hline Total & 100.0 \\
\hline
\end{tabular}

Estas respuestas aportan evidencia de que, en su mayoría (70 \%), los procesos de gestión académica que desempeñan personas con puestos de gestión en el CIDE son concebidos como no saludables, como una carga que genera estrés y que limita el crecimiento personal. Parecen indicar, además, que la Universidad ha perdido el rumbo y se ha burocratizado, aspecto que es necesario reestablecer desde una visión y actuación que potencializa los entornos saludables. Según San Nicolás (2014):

El concepto de entorno de trabajo saludable ha evolucionado en los últimos años desde un enfoque casi físico de trabajo, a un esquema más amplio de seguridad y salud que se amplía hasta incluir los hábitos de salud, estilos de vida, y factores psicosociales (organización del trabajo, riesgos emergentes y cultura de trabajo). (p. 32) 
La opinión de las personas entrevistadas hace referencia, precisamente, a una multiplicidad de factores tanto institucionales como personales que les hace considerar su puesto saludable o no. Aspecto evidentemente complejo vinculado con las percepciones y construcciones mentales, patrones asumidos no como producto de una formación previa al puesto, sino como parte de la vivencia en la cotidianeidad, de ahí que, para algunas personas, el puesto es saludable dependiendo de la forma en que sea asumido.

Desde esta perspectiva, es importante destacar que las personas que respondieron que su puesto sí es saludable (30\%), lo justifican por la forma y la actitud en que ellos y ellas, como personas, manejan y asumen las responsabilidades derivadas de este, así como las prioridades; destacando que se debe estar preparado para asumir el puesto y capacitarse para hacer un cambio total de vida.

Lo anterior hace reflexionar a las investigadoras sobre que, para asegurar que una gestión universitaria sea saludable, es necesario garantizar un proceso formativo que permita comprender y dar sentido a la cultura institucional, dinamizando sus estructuras y formas de trabajo. Esto concuerda con lo planteado por Basora y León (2013):

Es necesario entender que la cultura universitaria, es decir, el sistema de valores, ideas y normas que comparten los actores, obedece a la interacción de los procesos de formación universitaria, la gestión, las capacidades, la voluntad, la estructura y los procesos de la organización y el entorno superior en el que vive la organización por ser en esas interrelaciones donde se transforma y desarrolla la formación y la identidad en la búsqueda constante de la pertinencia social. (p. 39)

Otros aspectos vinculados con la investigación se relacionan con las condiciones laborales en el ámbito institucional y salud, específicamente en la unidad académica donde cada persona con puesto de autoridad labora. Las preguntas para indagar sobre estos aspectos fueron:

\section{¿Cuán saludable es su unidad académica o departamento?}

El 50\% de las personas participantes considera que mucho, 30\% opina que medianamente y $20 \%$ cree que es poco saludable.

Quienes opinan que el lugar donde trabajan es muy saludable, hacen referencia a la cohesión de grupo, que se comparte la reflexión y al compromiso del equipo de trabajo, así como a una cultura organizacional participativa y afectiva, con relaciones horizontales, donde prevalece un ambiente de buenas relaciones y de disfrute. Indican que en su unidad académica no se confunde el trabajo con la vida personal y que se tiene apoyo institucional para el manejo del estrés y el desarrollo de la inteligencia emocional. 
doi: http://dx.doi.org/10.15359/ree.19-3.2

URL: http://www.una.ac.cr/educare

CORREO: educare@una.cr

Una persona expresa: "Es una Unidad Académica o departamento, es muy alegre, muy de compartir, eso le da que sea saludable. Es una Unidad Académica de reflexión. Uno disfruta trabajando en esta Unidad Académica".

Las personas que consideran medianamente saludable su unidad académica, aluden a luchas de poder, confrontaciones o conflictos entre personas o carreras, así como a falta de madurez de los otros y otras. También hacen referencia a sobrecarga de trabajo y estrés generado por las dinámicas institucionales. Todo ello al lado de flexibilidad en los procesos, autosatisfacción en el trabajo y que se cuenta con espacios para compartir. Una de las autoridades indica que "La dinámica institucional somete a situación de estrés. Hay aspectos positivos de autosatisfacción".

El restante $20 \%$ de las autoridades del CIDE considera que su lugar de trabajo es poco saludable. Sus argumentos al respecto hacen referencia a falta de visión de equipo y de compartir, falta de apropiación de la misión y visión de la unidad académica de parte de algunas y algunos funcionarios y no distinción entre lo personal y laboral. Una persona manifiesta: "Tenemos partes enfermas".

Lo anterior se clarifica con figura 2:

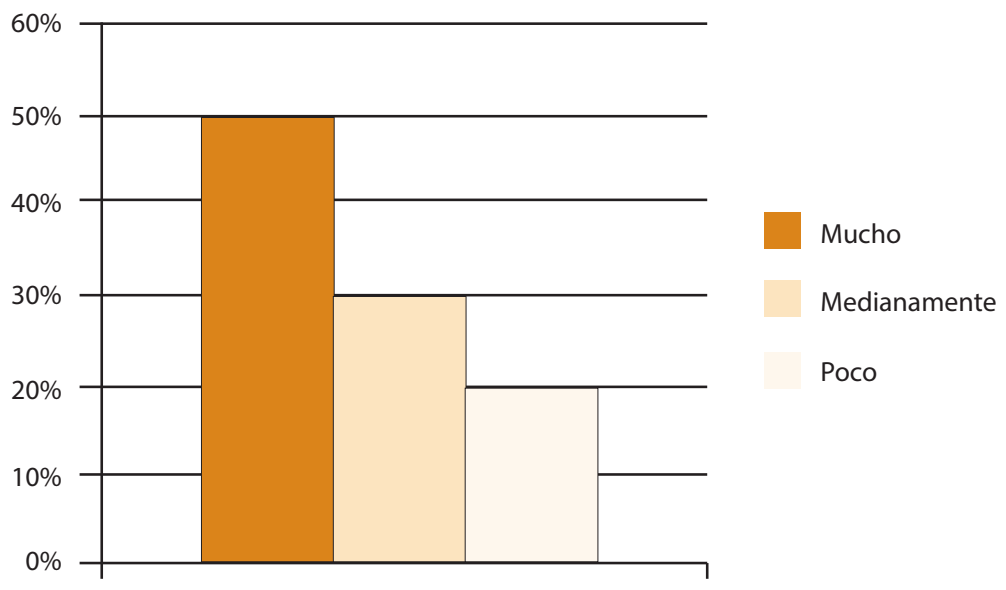

Figura 2. Cuán saludable es la unidad académica, según criterio de las autoridades del CIDE.

No obstante los resultados anteriores, resulta interesante contrastar la percepción de unidad académica saludable que manifiestan autoridades que están frente a una misma unidad académica ya que, de las cuatro unidades que tienen puestos en dirección y subdirección, solamente en una de ellas se encontró concordancia al respecto, en las restantes tres unidades académicas hubo diferencia de criterios, lo que se detalla en la tabla 4. 
Tabla 4

Coherencia entre autoridades de la misma unidad académica respecto a lo saludable que consideran su unidad académica o departamento

\begin{tabular}{cll}
\hline \multirow{2}{*}{ Unidad Académica } & \multicolumn{2}{c}{ Cuán saludable es su unidad académica } \\
\cline { 2 - 3 } & Dirección & Subdirección \\
\hline Unidad 1 & Muy saludable & Medianamente saludable \\
Unidad 2 & Medianamente saludable & Muy saludable \\
Unidad 3 & Medianamente saludable & Poco saludable \\
Unidad 4 & Muy saludable & Muy saludable \\
\hline
\end{tabular}

Esta comparación permite deducir que la percepción que tenga una autoridad, respecto a lo saludable que es la unidad académica en la que tiene un puesto de conducción, es un criterio con matices subjetivos que no depende enteramente ni de las exigencias del puesto (dirección o subdirección) ni de la complejidad de la unidad académica y, como fue indicado anteriormente y fue mencionado por una tercera parte de las autoridades entrevistadas, la apreciaciones están relacionada con la actitud y la manera de asumir el puesto.

\section{¿Considera que su puesto en la Universidad es saludable?}

Un 70 \% señala que no, mientras que un 30 \% expresa que sí. Esta información se correlaciona con lo expuesto en respuestas anteriores, donde se explicitan los aspectos de orden funcional y burocráticos que demanda el puesto; exigencia de más tiempo para asumir gran cantidad de tareas y dejar de lado aspectos relevantes de la vida universitaria.

\section{Identificación de experiencias saludables concretas que impulsa la persona entrevistada}

Loanteriorse puedetambién percibirenlarespuestaala pregunta quesugierelaidentificación de experiencias saludables concretas que impulsa, en su calidad de autoridad o puesto de gestión en la unidad académica o departamento; el $10 \%$ indica que no promueve ninguna experiencia saludable. Un comentario al respecto es que "No hay políticas ni en la Unidad ni en el Centro. Nunca se tienen espacios para el esparcimiento. Sólo resolver y no convivir". Evidentemente esto permite definir la importancia de identificar opciones laborales que contribuyan a construir experiencias donde se puedan expresar no solo conocimiento, sino emociones y pasiones. Un reto evidente y necesario que debe asumirse desde la postura de políticas claras. 
doi: http://dx.doi.org/10.15359/ree.19-3.2

URL: http://www.una.ac.cr/educare

CORREO: educare@una.cr

Por otra parte, el 90\% exterioriza que impulsa experiencias saludables en su unidad académica, circunscritas al estudiantado, sector administrativo o académico. En relación con el estudiantado, se hace referencia a procesos de inducción e información para la inserción positiva en la universidad y el manejo del estrés, apoyo y orientación del profesorado, buen servicio administrativo para atender los trámites estudiantiles, presencia de autoridades de unidad académica en las aulas para promover la participación estudiantil, vinculación con las asociaciones de estudiantes y representantes de grupos, giras con grupos de estudiantes y convivio de estudiantes y docentes.

En este sentido, una persona expone:"Con respecto a los estudiantes, se generan procesos de inducción e información para la inserción más positiva y manejo de la ansiedad. Se les ofrece apoyo de un consejero, profesor orientador por nivel que tiene cercanía con el estudiante. Hay coordinador a nivel administrativo".

Acerca del personal administrativo se mencionan actividades que promueven familiaridad y espacios para compartir. También que se generan espacios para atender la salud física, se promueven giras para que el sector administrativo conozca realidades académicas. En este sentido, se afirma que: "En lo administrativo se ha conformado como equipo de trabajo un grupo muy familiar, como apoyo. Las reuniones administrativas son espacios para que todos opinen cómo se sienten, cómo mejorar y dividir el trabajo".

Las experiencias saludables que realizan las autoridades del CIDE con el sector académico, según su opinión, buscan generar espacios de diálogo y de compartir. Es así como se menciona la realización de celebraciones, actividades sociales y poner en común la producción académica. Se pretende encauzar el debate académico en un ambiente agradable. Una de las personas entrevistadas comenta que:"Respecto a los académicos, estamos proponiendo un plan de capacitación, trabajo en equipo, relaciones interpersonales. Estamos trabajando la visión de una unidad".

\section{Visualización de una pedagogía saludable}

Fue también interés de esta investigación, conocer la forma en la que las autoridades que lideran los procesos pedagógicos y administrativos del CIDE visualizan una pedagogía saludable, constructo teórico en construcción, aporte relevante e innovador de esta investigación.

Se obtuvieron 24 respuestas, las cuales fueron agrupadas según fue ofrecida una definición del término, una caracterización o una forma de llevar a cabo una pedagogía saludable.

La mayoría de las respuestas se ubicó entre ofrecer una definición de lo que las autoridades consideran es una pedagogía saludable (41.7\%) y la forma en que consideran que esta puede ser trabajada en el aula universitaria y, en algunos casos, en la cotidianidad misma de la vida del ser humano (41.7\%). 
Un porcentaje menor (16.6 \%) incluyó algunas características de lo que considera o visualiza es una pedagogía saludable.

La tabla 5 transcribe de manera sistematizada la totalidad de respuestas obtenidas a partir del constructo pedagogía saludable, caracterización e implementación. De acuerdo con lo anterior, se establecen las siguientes categorías.

Tabla 5

Representaciones de las personas que lideran el CIDE con respecto a la pedagogía saludable, su caracterización, e implementación. Agrupado por categorías

\begin{tabular}{|c|c|c|}
\hline Categoría de análisis & Respuestas obtenidas & Porcentaje \\
\hline $\begin{array}{l}\text { Definición de pedagogía } \\
\text { saludable }\end{array}$ & $\begin{array}{l}\text { Ve el ser como totalidad. } \\
\text { - Es un modelaje como parte de una comunidad. } \\
\text { - Es vivencia y actitud hacia la vida que contempla al ser humano como una } \\
\text { totalidad en su relación con el otro u otra. } \\
\text { - Es expresión de amorosidad en su relación con el otro u otra. } \\
\text { - Procesos de comunicación abierta, respetuosos y buscar relaciones } \\
\text { horizontales. } \\
\text { - Una que tome al ser humano como centro / pensada en la persona como ser } \\
\text { integral. } \\
\text { - En la que se tome la educación como proceso personal e integral. } \\
\text { - Es una práctica de vida. } \\
\text { - Es una filosofía de vida que puede transmitirse a las demás personas. } \\
\text { - Aquella que busca generar equilibrio entre lo académico y lo personal. } \\
\text { - Incluye valores como el amor, cooperación, solidaridad y empatía, en el } \\
\text { quehacer cotidiano. } \\
\text { - Se da en la convivencia. } \\
\text { - Son procesos respetuosos y horizontales. } \\
\text { Hay disposición a escuchar. }\end{array}$ & 41.7 \\
\hline $\begin{array}{l}\text { Caracterización de } \\
\text { pedagogía saludable }\end{array}$ & $\begin{array}{l}\text { Debería de ser una filosofía de los cursos que hay que transversalizar. } \\
\text { - Hay que definir salud y transversalizarlo en los cursos. } \\
\text { - Hay que incluirla en actividades diarias. } \\
\text { - Que los estudiantes sepan que cada docente, ejerce una función facilitadora } \\
\text { y que también está aprendiendo. }\end{array}$ & 16.6 \\
\hline $\begin{array}{l}\text { Cómo llevar a cabo una } \\
\text { pedagogía saludable }\end{array}$ & $\begin{array}{l}\text { Con la persona guía académica y coordinadora de nivel. } \\
\text { - Trabajando autoestima unido a la materia. } \\
\text { - Formación para atender hábitos que permitan un mejor desarrollo del } \\
\text { cuerpo. } \\
\text { - Dando talleres e incorporando contenidos sobre relaciones interpersonales, } \\
\text { manejo del estrés y del tiempo, valores, ética. } \\
\text { - Primero hay que empoderarse. } \\
\text { - Conociendo al grupo, las historias de vida del estudiantado. }\end{array}$ & 41.7 \\
\hline Total & & 100.0 \\
\hline
\end{tabular}


doi: http://dx.doi.org/10.15359/ree.19-3.2

URL: http://www.una.ac.cr/educare

CORREO: educare@una.cr

Como se observa, dentro de la categoría "definición" de lo que visualizan las autoridades del CIDE como una pedagogía saludable, incluye aspectos vinculados con la integralidad del ser humano y de su relación con una comunidad y como parte de ella. Además del equilibrio entre lo académico y lo personal, la comunicación abierta y la búsqueda de relaciones horizontales. Identifican por otra parte la pedagogía saludable como una filosofía de vida en la que la relación con el otro u otra sea expresión de amor.

La categoría "cómo se lleva a cabo este tipo de pedagogía" se describe en respuestas tales como: salud debería definirse y realizar un trabajo de transversalización en las carreras; incluir talleres, charlas sobre relaciones interpersonales, manejo del estrés y del tiempo, así como valores y ética, entre otros. Lo anterior, sin dejar de lado respuestas que indican que el personal docente debe ser percibido por el estudiantado como un facilitador del proceso en el que también está aprendiendo.

Finalmente, entre quienes se agrupan en la categoría "caracterización" de la pedagogía saludable mencionaron procesos horizontales y respetuosos; disposición a la escucha y la existencia de valores en la cotidianidad.

\section{¿Qué experiencias saludables se desarrollan en el aula en su unidad académica o departamento?}

Para efectos de identificar experiencias saludables concretas según la opinión de quienes participaron se planteó la pregunta y se pidió citar experiencias docentes concretas.

Las respuestas fueron agrupadas en tres categorías. En la primera, correspondiente a un 51.7 \% de las respuestas, se integran las vinculadas con aspectos de índole "socio-afectivo" que incluyen experiencias participativas que permiten el autoconocimiento, el conocimiento de otras personas y del entorno social y cultural. Una segunda categoría que abarca el $34.5 \%$ de las respuestas, se ubica en un plano más "académico y curricular", describe experiencias vinculadas con componentes temáticos y cognoscitivos. Las respuestas restantes que corresponden al 13.8 $\%$, destacan la ausencia de experiencias saludables en las aulas de su unidad académica y se concentra en mencionar "otras percepciones" que considera poco saludables.

La tabla 6 presenta de manera sistematizada las respuestas obtenidas a partir de la voz de las autoridades que lideran procesos académicos y administrativos en el CIDE.

Es importante señalar que, como se observa en la tabla 6, dentro de las experiencias saludables mencionadas por las personas participantes, se hace alusión a espacios externos al aula como las giras, los paseos, los juegos lúdico-creativos y los convivios, aspectos que han sido mencionados en otras respuestas de la entrevista. Esto debería ser un análisis a lo interno del Centro de Investigación y Docencia en Educación y en la Universidad Nacional, comprendiendo que los procesos educativos y las experiencias pedagógicas tienen sentido cuando se insertan en la realidad y se asocian a la vida misma. 
doi: http://dx.doi.org/10.15359/ree.19-3.24

URL: http://www.una.ac.cr/educare

CORREO: educare@una.cr

Tabla 6

Experiencias saludables concretas que se desarrollan en el aula, según opinión de las personas que lideran procesos académicos y administrativos categorizados por áreas

\begin{tabular}{|c|c|c|}
\hline Categoría & Experiencias saludables & Porcentaje \\
\hline $\begin{array}{l}\text { Área } \\
\text { Socio-afectiva }\end{array}$ & $\begin{array}{l}\text { - Acompañamiento a estudiantes } \\
\text { - Preocupación de profesorado por el ser humano } \\
\text { - Actividades pensadas en el bienestar estudiantil } \\
\text { - Convivios } \\
\text { - Cursos en que estudiantes se conozcan primero a ellos mismos o a ellas } \\
\text { mismas como seres aprendientes } \\
\text { - Viajes introspectivos para resolver asuntos personales } \\
\text { - Conocimiento de sí y de las otras personas } \\
\text { - Historias de vida, cercarse a las personas } \\
\text { - Hacer convivios al final del curso } \\
\text { - Celebrar los cumpleaños } \\
\text { - Talleres como espacio para crecer } \\
\text { - Acciones de solidaridad } \\
\text { - Búsqueda de bienestar estudiantil } \\
\text { - Trabajar áreas como autorrealización profesional, proyecto de vida, metas y } \\
\text { objetivos } \\
\text { - Crecer como personas y desarrollar sensibilidad social como docentes }\end{array}$ & 51.7 \\
\hline $\begin{array}{l}\text { Área } \\
\text { Académica y curricular }\end{array}$ & $\begin{array}{l}\text { - Giras pedagógicas para conocer otras realidades educativas } \\
\text { - Salir del aula es saludable, hacer cambios y reuniones fuera de la universidad } \\
\text { - Actividades culturales y lúdicas } \\
\text { - Proyectos que favorezcan del rendimiento académico } \\
\text { - Cursos específicos (Educación para la salud y calidad de vida; Desarrollo } \\
\text { personal y comunicación humana) } \\
\text { - Cursos que desarrollan un trabajo muy equilibrado desde nutrición, estética } \\
\text { y que organicen sus procesos } \\
\text { - Cursos vinculados con proyectos como pedagogía social } \\
\text { - Cursos en que se trabaje en la estrategia de talleres } \\
\text { - Proyectos y actividades de extensión que involucren visitas a hospitales y el } \\
\text { trabajo con personas adultas mayores y personas con discapacidad } \\
\text { - Adecuaciones en los cursos }\end{array}$ & 34.5 \\
\hline Otras percepciones & $\begin{array}{l}\text { Estamos acostumbrados al tema de conocimientos, cursos que no } \\
\text { promueven el movimiento } \\
\text { En la soda no se puede almorzar en paz, no es posible disfrutar el almuerzo } \\
\text { por el ruido } \\
\text { - Parte del estudiantado, por el traslado o por falta de dinero, no puede comer } \\
\text { a sus horas } \\
\text { - No hay valoración del cuerpo humano como tal }\end{array}$ & 13.8 \\
\hline Total & & 100.0 \\
\hline
\end{tabular}


doi: http://dx.doi.org/10.15359/ree.19-3.2

URL: http://www.una.ac.cr/educare

CORREO: educare@una.cr

\section{Reflexiones generales y aportes de este artículo de investigación}

Las investigadoras resaltan que es imprescindible que la Universidad Nacional de Costa Rica, piense, se organice y gestione, propiciando ambientes mentales y contextuales saludables. El Centro de Investigación y Docencia en Educación como formador de formadores y formadoras, y cuna para la formación de la docencia universitaria, debe posicionar la pedagogía como ciencia de la vida que investiga y analiza los procesos educativos para transformarlos en mejoras cualitativas y cuantitativas personales y sociales.

La vida universitaria tiene que ofrecer oportunidades de crecimiento cognoscitivo, socioafectivo, psicomotriz, integrando, indudablemente, valores y principios que permitan el cultivo del afecto y del espíritu. No se pueden gestionar procesos académicos y administrativos si no se parte de que la pedagogía tiene el papel de formar para la vida, para una ciudadanía planetaria que exige comprender que la salud es uno de los propósitos que deben guiar las acciones educativas, porque la salud es un elemento esencial para que la inteligencia en su máxima expresión fluya. "La principal fortaleza de una organización exitosa, es: el personal y la nueva filosofía del trabajo, en la que los valores humanos son imprescindibles al formar parte de la vida y la cultura organizacional" (Soberanes y de la Fuente, 2009, p. 127).

Las nuevas tendencias en la gestión del talento humano aprecian que cuando las personas se sienten motivadas a nivel personal y colectivo existe un espíritu de compromiso y de identificación que rinde mejores y mayores resultados conceptuales, procesuales y actitudinales. Es necesario potenciar políticas que evidencien la valoración y el respeto a la vida en sus diferentes dimensiones y manifestaciones, y la salud debe ser atendida como ese derecho fundamental que transversa y permea todo el proceso pedagógico para lograr que haya gestión de vida.

Evidentemente, la investigación permite como hallazgo destacar que las percepciones que tengan las personas que conviven y lideran procesos universitarios con respecto a la salud, el cuidado, el bienestar y la calidad de vida, se entrelazan de manera recursiva con la vertiente colectiva en la construcción de sentidos, y se forjan mutuamente. Es necesario resignificar y redignificar el sistema de gestión universitaria como un sistema, un organismo vivo donde convive y coexiste una diversidad de diversidades.

Las calidades de los proceso socioeducativos a nivel universitario no solamente se miden por indicadores cuantitativos, sino que es de especial atención los aspectos de índole cualitativo que no pueden ser abordados desde paradigmas mecanicistas.

La universidad forma seres humanos para la vida, por tanto, tiene que preocuparse por generar espacios para fortalecerla integralmente. Desde un paradigma de la complejidad, donde se valoran las redes y los vínculos, es innegable que la Universidad debe ir cerrando brechas. 
Los resultados académicos o el seguimiento a normativas prescriptivas no son evidencia de que la institución está siendo promotora de vida, ni de los procesos auténticos de aprendizaje significativo y relevante para las personas que conviven en ella.

Toda organización como organismo vivo se mueve a partir de redes de apoyo y se fortalece a partir de experiencias gratificantes que permiten mantener vigente la emoción de aprender, de convivir, de hacer y de ser.

\section{Conclusiones y construcciones relevantes de la investigación al campo del conocimiento}

Las nuevas exigencias y demandas obligan a las organizaciones a buscar mecanismos para visualizarse como un sistema cuya sinergia hace que el funcionamiento de la institución sea exitoso a partir de la comprensión compartida de todas las personas y de los procesos de cambio propios de sociedades complejas.

En este sentido, la Universidad Nacional de Costa Rica y el Centro de Investigación y Docencia en Educación deben hacer lectura permanente de los cambios, para ser coherentes en sus formas de organización administrativa, académica y estudiantil, sugiriendo experiencias variadas y polivalentes que permitan ir en consonancia con las necesidades nacionales e internacionales. Desde luego, comprendemos que este es un proceso complejo, e incierto, que implica un cambio en la ruta de abordaje, por tanto, un cambio paradigmático. En este se aprecia que no solo lo medible y cuantificable es lo valioso, sino que desde un paradigma emergente se retoma la esencia de la vida como elemento dinamizador sociohistórico.

Las instituciones tienen vida a partir de los pensares, sentires y actuares de sus actoras y actores: estudiantes, personal académico y personal administrativo. Es necesario trascender el paradigma lineal y jerárquico, para comprender que la Universidad es una comunidad aprendiente y holística donde la suma de particularidades, bien mediadas, generan un "todo" con sentido humano vital, lo cual contradice las tendencias neoliberales y permite descubrir el potencial que se tiene para contraponerse a las políticas de mercado de consumo que mercantilizan la educación, anulan la creatividad y dejan de lado la vida y formación del ser en su asombrosa amplitud.

Las universidades requieren desarrollar una ética de cuidado profundo, fomentando estilos de vida basados en la comunicación dialógica y dialogante con la diversidad; aspecto que exige respeto, solidaridad, interpretación y compasión para asumir la realidad y sus variables. Un ser humano saludable en el amplio sentido del término es capaz de aportar no solo a la fuerza laboral y económica de un contexto, sino que es capaz de cambiar el entorno a partir de una visión humanizadora, y su actuación humanizante, lo cual se vincula con reconocimiento de los derechos fundamentales, pero también con responsabilidades mundiales y universales. 
doi: http://dx.doi.org/10.15359/ree.19-3.2

URL: http://www.una.ac.cr/educare

CORREO: educare@una.cr

En la medida en que se reconozca que los entornos saludables generan vidas saludables, las instituciones educativas serán capaces de planificar innovaciones educativas fundadas en el respeto y en la legitimación de la pluralidad que emerge del sentir y del pensar; principios y complementos vitales para cualquier progreso pedagógico.

Como parte del proceso de investigación, desarrollado en la Universidad Nacional de Costa Rica y preocupadas por la pedagogía y sus necesarias rutas de abordaje, descubrimos que podemos aproximarnos a rutas alternativas y creativas para generar procesos pedagógicos que, basados en principios y valores, enriquezcan y enaltezcan la vida. Lo anterior conlleva procesos de interaprendizaje; contemplación y resignificación de las diferencias como requisito para enriquecer reflexiones, interpretaciones y acciones que iluminen una forma de gestión académica universitaria más vigorosa, aspecto asociado fuertemente con el concepto acuñado por el equipo de investigación sobre pedagogía saludable.

Una gestión académica saludable, entonces, conlleva implícito el concepto de innovación, de aprendizaje permanente y de la adecuada apropiación de los cambios. Una institución que privilegia el humanismo como visión debe estar abierta siempre a la innovación, promoviendo un estilo de vida institucional saludable.

Evidentemente el camino es arduo, es necesario hacer rupturas mentales que permitan fomentar una gestión académica que favorezca la motivación y el aprendizaje, el amor por la vida. Una universidad saludable necesariamente se compromete con el desarrollo vital. Ese que vincula mente, cuerpo y espíritu y lo mantiene en armonía con el contexto. Desde luego, esta es una función social que involucra otros ámbitos, actores y, ante todo, la convicción personal de que la vida es el mayor tesoro por lo cual hay que asegurar el derecho a la salud y a una educación que la enaltece.

Generar experiencias saludables, desde una pedagogía saludable, alude a que la universidad y el conjunto de instancias y personas que la conforman deben profundizar en la práctica de experiencias que permitan concebir un ambiente de libertad, de democracia, de diálogo, de reflexión crítica y de compromiso ético.

El establecimiento de un estilo de vida saludable en la universidad pasa por las creencias de cada una de las personas de la comunidad universitaria y quienes tienen a su cargo la gestión del quehacer requieren tener conciencia de las repercusiones de sus ideas y de sus conductas en el colectivo.

\section{Resaltando los aportes de participantes}

De las sugerencias ofrecidas por las personas entrevistadas, se tienen conclusiones y hallazgos relevantes para esta investigación, entre las que destacan: 
Para vivir una pedagogía saludable es necesaria la ruptura de la visión de CIDE como la suma de unidades académicas o departamentos, para construir colectivamente una visión de Centro que promueve los derechos humanos.

Se sugiere la necesidad de contar con el diseño de políticas que puedan ser consideradas y abordadas desde cada unidad como un todo y por qué no como gestores de procesos formativos integrar e irradiar hacia toda la comunidad universitaria y con esto impactar la realidad nacional.

Es necesario que la pedagogía saludable se encuentre visibilizada en los cursos de educación permanente y continua que se desarrollan en el CIDE, para proyectar su vivencia a nivel del centro, la universidad, la comunidad o comunidades que están representadas.

Se requiere establecer políticas universitarias claras que fomenten la alfabetización en cuanto a lo que significa la pedagogía saludable y su apropiación. De forma que se fomenten espacios de aprendizaje más asertivos y sensibles.

Para lograr un proceso pedagógico saludable es necesario reconocer la diversidad de contextos y de personas que conviven. El respeto y el reconocimiento de la otredad, es vital.

Es importante disponer de espacios de infraestructura y tiempos que motiven la reflexión académica e incorporen lo urgente de reconocer y actuar sobre los nuevos escenarios sociohistóricos que nos desafían y exigen hacer algo más por la vida en todas sus manifestaciones.

El trabajo articulado conlleva aprender a entender, a compartir, a promover y a ser agentes de vida saludable; implica un cambio personal y colectivo, donde se rescata la cultura de los afectos vinculada con los conocimientos. Es decir, se comprende que la pedagogía es vida y que protegerla es una decisión y un proceso de gestión colaborativo.

\section{Referencias}

Ardila, R. (2003). Calidad de vida: Una definición integradora. Revista Latinoamericana de Psicología, 35(2), 161-164. Recuperado de http://www.redalyc.org/pdf/805/80535203.pdf

Assmann, H. (2002). Placer y ternura en la educación. Hacia una sociedad aprendiente. Madrid: Narcea.

Basora, O. C. y León, R. (Octubre-Diciembre, 2013). La gestión de las instituciones de educación superior en la formación de la pertinencia e identidad universitaria. Revista Didasc@ lia: Didáctica y Educación, 4(4), 31-44. Recuperado de http://ojs.uo.edu.cu/index.php/ Didascalia/article/view/3650 
doi: http://dx.doi.org/10.15359/ree.19-3.2

URL: http://www.una.ac.cr/educare

CORREO: educare@una.cr

Castillo, I., Castillo, R., Flores, L. E. y Miranda, G. (2013). Proyecto pedagogía saludable en el CIDE. Heredia, Costa Rica: Universidad Nacional de Costa Rica, CIDE.

Castillo, I., Castillo, R., Flores, L. E. y Miranda, G. (Mayo-Agosto, 2014). Pedagogía saludable: Despertar de un nuevo nodo. Revista Electrónica Educare, 18(2), 311-320. doi: http://dx.doi. org/10.15359/ree.18-2.16

Domínguez, L. R., Ramírez, A. F.y García, A. (Enero-Junio, 2013). El clima laboral como elemento del compromiso organizacional. Revista Nacional de administración, 4(1), 59-70. Recuperado de http://dialnet.unirioja.es/servlet/articulo?codigo $=4721419$

Dossey, L. (1999). Tiempo, espacio y medicina (3 ed.). Barcelona: Kairós.

Dossey, L. (2004). El poder curativo de la mente. La salud más allá del cuerpo. México: Santillana.

Gairín, J. (2010). La evaluación del impacto en programas de formación. Revista lberoamericana sobre Calidad, Eficacia y Cambio en Educación, 8(5), 19-43. Recuperado de http://www. rinace.net/reice/numeros/arts/vol8num5/art1.pdf

Gimeno, M.Á., Grandío, A.y Marqués, A. I. (Junio, 2013). El cambiantemundo de las organizaciones. Hacia una organización saludable. Revista Internacional de Organizaciones, 10, 41-63. Recuperado de http://www.revista-rio.org/index.php/revista rio/article/view/122/pdf

Gutiérrez, F. (1981). Educación como praxis política. México: Siglo XXI.

Hernández, C., Fernández, R. y Baptista, P. (2006). Metodología de la investigación (4ª ed.). México, D.F.: McGraw-Hill.

Maturana, H. (1996). El sentido de lo humano. Chile: Dolmen Ediciones. Recuperado de http:// escuelainternacionaldecoaching.com/downloads/BibliotecaEIC/Humberto $\% 20$ Maturana\%20-\%20El\%20Sentido\%20de\%20lo\%20Humano.pdf

Maturana, H. (1999). Transformación en la convivencia. Santiago de Chile: Dolmen Ediciones.

Maturana, H. y Varela, F. (1986). El árbol del conocimiento. Las bases biológicas del entendimiento humano. Santiago de Chile: Editorial Universitaria.

Morin, E. (2001). Los siete saberes necesarios para la educación del futuro. Barcelona: Paidós.

Najmanovich, D. (Enero, 2001). Pasos hacia un pensamiento complejo en salud. Ponencia presentada en el Primer Seminario Bienal de Implicaciones Filosóficas de las Ciencias de la Complejidad. La Habana, Cuba. Recuperado de http://www.denisenajmanovich.com. ar/htmls/0303 textos.php 
Payán, J. C. (2000). Lánzate al vacío. Se extenderán tus alas. Bogotá: McGraw-Hill.

San Nicolás, M. (Enero, 2014). Construyendo un entorno de trabajo más seguro y saludable. Capital humano, 27(283), 32-34.

Soberanes, L. T. y de la Fuente, A. H. (Julio-Diciembre, 2009). El clima y el compromiso organizacional en las organizaciones. Revista Internacional La Nueva Gestión Organizacional, 5(9), 120-127. Recuperado de http://www.uaeh.edu.mx/investigacion/icea/LI GestEmp/ lucia sob/2.pdf

Vila, A., Escotet, M. A. y Goñi, J. J. (2009). Elementos para un modelo de innovación de las instituciones de educación superior. En N. Fernández (Comp.), Universidad, sociedad e innovación. Una perspectiva internacional (pp.133-136). Buenos Aires: Editorial de la Universidad Nacional de Tres de Febrero.

\section{(0) Cómo citar este artículo en APA:}

Castillo-Cedeño, I., Flores-Davis, L. E. y Miranda-Cervantes, G. (Setiembre-Diciembre, 2015). Gestión académica saludable en el contexto universitario. Revista Electrónica Educare, 19(3), 1-25. doi: http://dx.doi.org/10.15359/ree.19-3.24

Nota: Para citar este artículo en otros sistemas puede consultar el hipervínculo "Como citar el artículo" en la barra derecha de nuestro sitio web: http://www.revistas.una.ac.cr/index.php/EDUCARE/index 